\title{
Effects of Teriparatide on Treatment Outcomes in Osteoporotic Hip and Pelvic Bone Fractures: Meta-analysis and Systematic Review of Randomized Controlled Trials
}

\author{
Nam Hoon Moon, MD, Jae Hoon Jang, MD*, Won Chul Shin, MD, $\mathrm{PhD}^{+}$, Seok Jin Jung, MD $^{*}$ \\ Department of Orthopaedic Surgery, Bio-medical Research Institute, Pusan National University Hospital, \\ Pusan National University School of Medicine, Busan, Korea, \\ Department of Orthopaedic Surgery and Trauma Center, Pusan National University Hospital, Busan, Korea*, \\ Department of Orthopaedic Surgery, Pusan National University Yangsan Hospital, \\ Pusan National University School of Medicine, Yangsan, Korea ${ }^{+}$, \\ Department of Orthopaedic Surgery, Pusan National University Hospital, Busan, Korea ${ }^{*}$
}

The primary objective of this study was to evaluate randomized controlled trials (RCTs) that have reported the effects of teriparatide on bone-healing in osteoporotic hip and pelvic bone fractures to determine the efficacy of teriparatide in lowering the rate of treatment failure. A total of 2,809 studies were identified using a comprehensive literature search (MEDLINE [n=1,061], Embase [ $n=1,395]$, and Cochrane Library $n=353]$ ). Five RCTs were included in the final analysis. Treatment failure rates at the last follow-up of osteoporotic hip and pelvic bone fractures between the teriparatide and control groups was the primary outcome. Treatment failure was defined as non-union, varus collapse of the proximal fragment, perforation of the lag screw, and any revision in cases due to mechanical failure of the implant during the follow-up period. The number of treatment failures in the teriparatide and placebo groups were $11.0 \%$ ( $n=20$ out of 181) and $17.6 \%$ ( $n=36$ out of 205), respectively. Although the rate of treatment failure in the teriparatide group was lower than that in the control group, this difference was not significant (odds ratio, 0.81 [95\% confidence interval, $0.42-1.53$ ]; $\mathrm{P}=0.16 ; \mathrm{I}^{2}=42 \%$ ). This metaanalysis did not identify any significant differences in the rate of treatment failure between the teriparatide and control groups at final follow-up. Based on these results, we believe that there is a lack of evidence to confirm efficacy of teriparatide in reducing treatment failures in osteoporotic hip and pelvic bone fractures.

Key Words: Teriparatide, Parathyroid hormone, Hip fractures, Pelvic bone fracture, Osteoporosis

Submitted: May 3, 2020 1st revision: June 24, 2020

Final acceptance: June 27, 2020

Address reprint request to

Nam Hoon Moon, MD

(https://orcid.org/0000-0001-9975-0992)

Department of Orthopaedic Surgery, Bio-medical Research Institute,

Pusan National University Hospital, Pusan National University

School of Medicine, 179 Gudeok-ro Seo-gu, Busan 49241, Korea

TEL: +82-51-240-7248 FAX: +82-51-247-8395

E-mail: namhoonmoondgmail.com
This is an Open Access article distributed under the terms of the Creative Commons Attribution Non-Commercial License (http://creativecommons. org/licenses/by-nc/4.0) which permits unrestricted non-commercial use, distribution, and reproduction in any medium, provided the original work is properly cited. 
Nam Hoon Moon et al. Teriparatide and Bone Healing: Meta-analysis and Systematic Review

\section{INTRODUCTION}

With an increase in the incidence of osteoporotic fractures owing to the growing elderly population, teriparatide (a form of human parathyroid hormone) is being widely prescribed by many orthopedic surgeons for the treatment of osteoporosis ${ }^{1,2}$. The intermittent administration of human parathyroid hormone, including teriparatice, promotes bone formation, owing to the anabolic window created by the difference in the levels of bone formation and bone resorption markers, which can improve mineral content, density, and bone strength ${ }^{3-6)}$. Teriparatide was the only United States Food and Drug Administration (FDA)-approved anabolic therapeutic option indicated in the management of osteoporosis until the recent availability of the FDA-approved romosozumab $^{7-9}$. The theoretical advantages of teriparatide in bone formation led many orthopedic surgeons to expect that it might have a positive effect on bone healing after fracture or fracture surgery.

Bone healing is accelerated upon treatment with the parathyroid hormone in animal models ${ }^{10-12}$. Some studies also report the bone-healing potential of teriparatide, when used in human subjects who have vertebral or lower limb fracture ${ }^{13-17}$. However, recently published results from randomized controlled trials (RCTs) have questioned the efficacy of teriparatide on bone healing, contrary to the available data ${ }^{18-20}$. Furthermore, there are debates over the appropriateness of using teriparatide to promote bone healing after fractures, owing to a lack of evidence confirming its ability to improve fracture healing ${ }^{13,21,22)}$.

Osteoporotic hip and pelvic bone fractures are common in the elderly and are responsible for high mortality rates owing to post-fracture immobilization ${ }^{23,24}$. Although substantial evidence indicating that teriparatide efficaciously improves bone healing is required to determine the most appropriate treatment strategy for reducing post-fracture mortality by improving bone healing and early mobilization, relatively few meta-analyses have addressed this topic. We hypothesized that teriparatide would lower the treatment failure rate in osteoporotic hip and pelvic bone fractures. Therefore, the primary objective of this study was to analyze the results of RCTs that have reported the bonehealing effect of teriparatide in osteoporotic hip and pelvic bone fractures to determine the efficacy of teriparatide in reducing treatment failure rates.

\section{MATERIALS AND METHODS}

\section{Search Strategy}

This study was conducted based on the guidelines of Preferred Reporting Items for Systematic Reviews and Meta-Analyses (PRISMA) statement and the Cochrane review method. To identify studies evaluating the effects of teriparatide on treatment outcomes in osteoporotic hip and pelvic bone fractures, all records until March 2020 in the PubMed, Embase, and Cochrane Library databases were searched by an independent medical librarian. There were no restrictions regarding language, publication year, nationality, or race in the search process. Search terms used in the subject headings, text words, and keywords fields included the following: "teriparatide" [MeSH] OR "human parathyroid hormone" [MeSH] OR "hPTH" [MeSH] OR "Forteo" [MeSH]) AND ("hip fracture" [MeSH] OR "femoral neck fracture" $[\mathrm{MeSH}] \mathrm{OR}$ "intertrochanteric fracture" [TW] OR "subtrochanteric fracture" [TW]. After the initial database screening, two researchers manually searched additional relevant studies. In this meta-analysis, only RCTs were included; non-randomized comparative experimental trials, comparative observational studies, case series, and case reports were excluded. When multiple studies were published by the same author or group of authors on the same subject, only the most recent article was included in this study. Enrolled studies in our meta-analysis were required to comprise at least two treatment arms: a teriparatide group and a placebo injection or no injection group (Fig. 1).

\section{Inclusion Criteria}

Eligibility was determined based on the PICOS criteria (population, intervention, comparator, outcomes, and study design). Population: patients who had an osteoporotic fracture of the hip or pelvis; intervention: patients who used teriparatide injection; comparator: patients who received no injection or a placebo injection (control group); outcomes: radiological assessments for non-union, revision, and implant failure, in which, at least one of the mentioned radiological measurements was employed; study design: two reviewers independently reviewed the titles and abstracts of RCTs and chose relevant studies for a full-text review. The articles to be included in the study were based on reviewers' consensus; there was no disagreement over literature selection between the reviewers. 


\section{Hip \& Pelvis}

Hip Pelvis 32(4): 182-191, 2020

\section{Data Extraction and Assessment of Methodological Quality}

Using a predefined data extraction form, two reviewers independently extracted the following data from the selected studies: first author, year of publication, study design, sample size, mean age of the patients, mean follow-up duration, diagnoses of hip fractures, fixation instruments used for surgery, treatment failure, and clinical outcome assessments. Two reviewers independently evaluated the methodological quality of each study using the Cochrane risk-of-bias tool for RCTs, documenting their potential for bias in selection, performance, detection, attrition, and reporting. Decisions were based on the reviewers' consensus. Disagreements were resolved by a third reviewer. Publication bias was visually and quantitatively assessed using funnel plots and Egger's regression test, respectively. Publication bias was considered to be absent if the funnel plot was symmetrical and the $P$-value was $>0.05$.

\section{Statistical Analysis}

Treatment failure at the last follow-up of osteoporotic hip and pelvic bone fractures between the teriparatide and control groups was the primary outcome. Treatment failure included non-union, varus collapse of the proximal fragment, perforation of the lag screw, and any revision owing to mechanical failure of the implant during the follow-up period. The odds ratio (OR) and 95\% confidence intervals $(\mathrm{CI})$ were com- puted for these categorical variables. Heterogeneity was assessed using the $\mathrm{I}^{2}$ statistic, in which $\mathrm{I}^{2}=25 \%$ was considered low heterogeneity, $50 \%$ was considered moderate, and $75 \%$ was considered high. When there was no statistical evidence of heterogeneity $\left({ }^{2}<50 \%, P>0.1\right)$, a fixed-effects model was adopted; otherwise, a random-effects model was chosen. Forest plots were used to illustrate the results of each study, the pooled estimate of effect, and the overall summary effect. Significance was set at $P<0.05$. All statistical analyses were conducted using $\mathrm{R}$ software version 3.6.3 (2020; The R Foundation for Statistical Computing, Vienna, Austria).

\section{RESULTS}

\section{Search Summary and Study-selection Process}

The outline of the study-selection process is presented in Fig. 1. A total of 2,809 studies were identified through literature searches of MEDLINE $(n=1,061)$, Embase $(n=$ 1,395), and the Cochrane Library ( $\mathrm{n}=353$ ). No additional studies were identified via a manual search and 906 duplicate studies were excluded. After screening the titles and abstracts, 1,880 studies were excluded. After conducting a full-text review of the remaining 23 studies, five studies were included in the final analysis ${ }^{18-20,25-27)}$.

The five studies included 181 cases in the teriparatide group and 205 cases in the placebo group (Table 1). The mean follow-up duration ranged from 3-19.5 months. Treatment out-

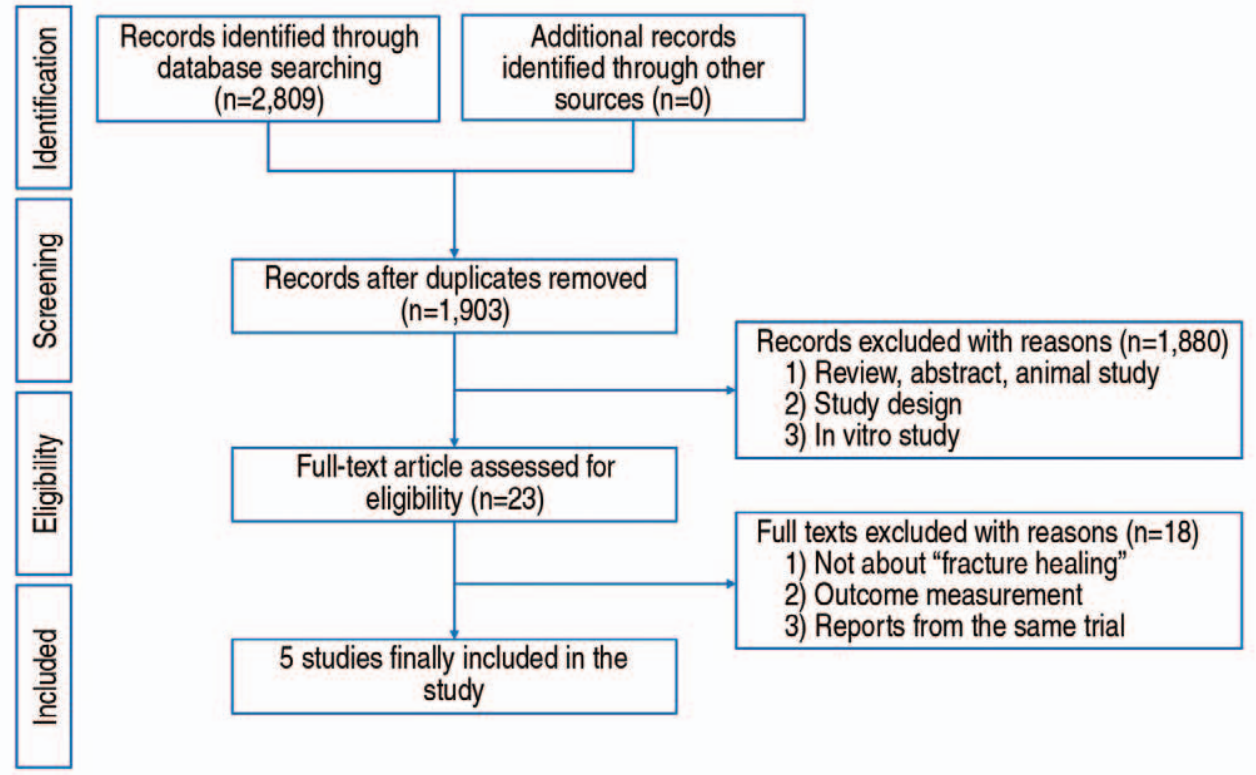

Fig. 1. PRISMA flow diagram. 
Hip \& Pelvis

Nam Hoon Moon et al. Teriparatide and Bone Healing: Meta-analysis and Systematic Review

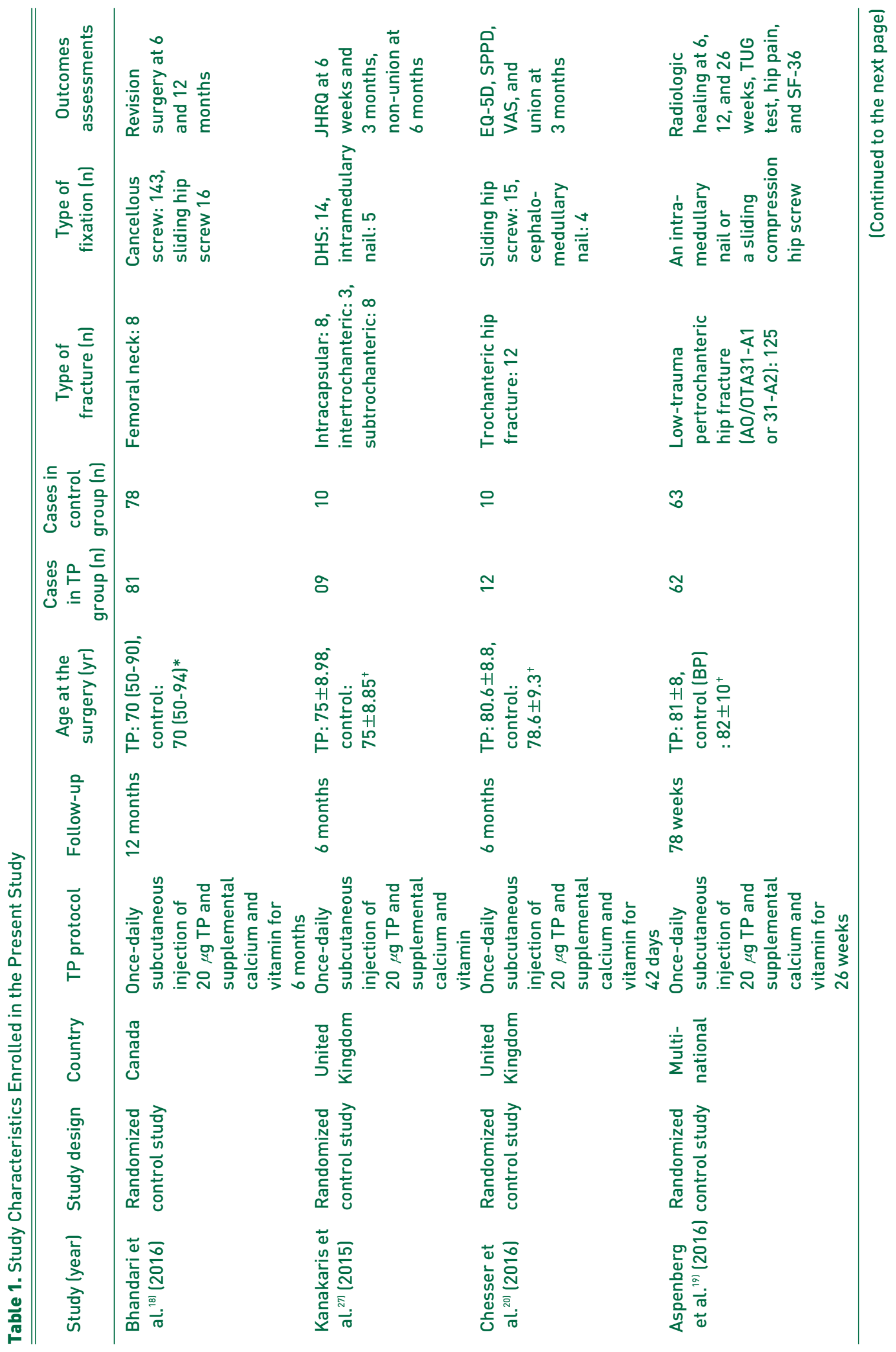




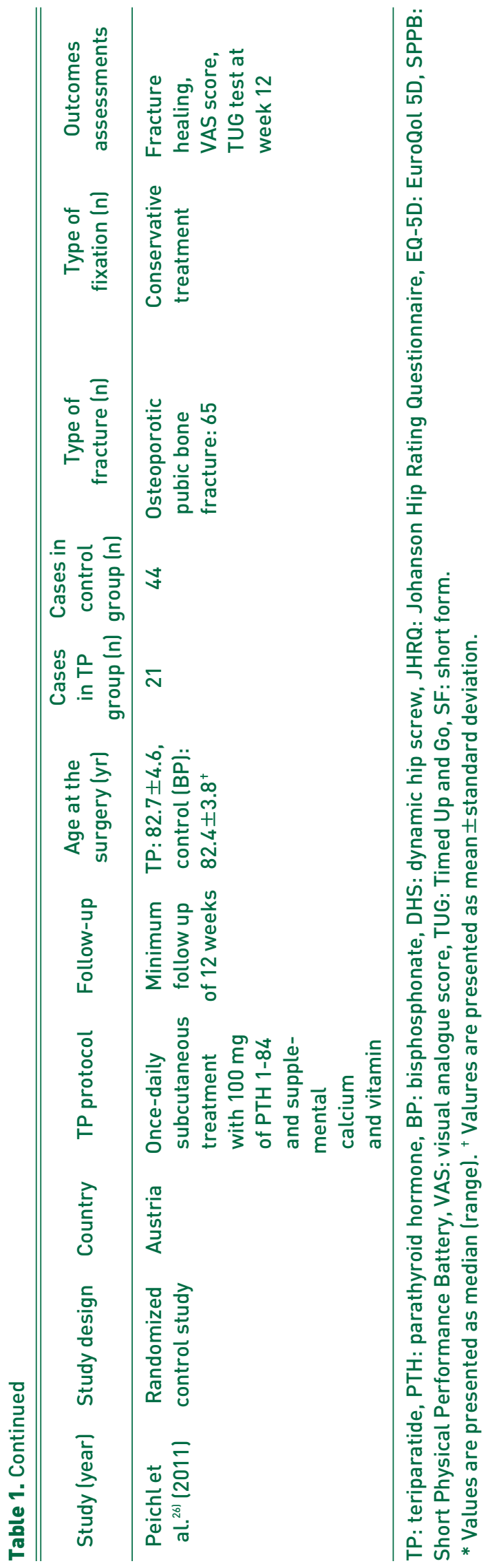

comes in these five studies included union rate, revision rate, mechanical failures, clinical scores, and complications. However, as radiological measurements and follow-up time points after surgery were different in each study, we assessed the rate of treatment failure at the last follow-up as the primary outcome.

\section{Pooled Estimate of the Effect of Postoperative Teriparatide}

A comparison of treatment failure rates in patients with osteoporotic hip and pelvic bone fracture between the teriparatide and placebo groups is presented in Table 2. For the analysis of treatment failures at the last follow-up, five studies comprising a total of 386 patients were assessed (teriparatide group $n=181$; placebo group $n=205$ ). Among the five included studies, four showed no significant difference in the rate of treatment failure between the teriparatide and control group; only Peichl et al. ${ }^{26)}$ demonstrated a lower rate of treatment failure in the teriparatide group compared with the control group. The total number of treatment failures included 20 out of 181 cases in the teriparatide group (11.0\%) and 36 out of 205 cases in the placebo group (17.6\%). Although the rate of treatment failure in the teriparatide group was numerically lower than that in the control group, this difference was not significant (OR, 0.81 [95\% CI, 0.421.53]; $P=0.16 ; \mathrm{I}^{2}=42 \%$ ) (Fig. 2).

\section{Risk-of-bias Assessment and Publication Bias}

Table 3 and Fig. 3 summarize the results of the risk-of-bias assessment of our meta-analysis. Asymmetry of funnel plots was suspected on visual assessment (Fig. 4). However, funnel plot asymmetry is generally considered as significant when more than 10 studies are included in a meta-analy$\mathrm{sis}^{28,29)}$. As only five studies were included in our meta-analysis, the number of studies was too small to be subjected to the Egger's regression test to conduct statistical analysis for asymmetry.

\section{DISCUSSION}

We conducted this meta-analysis of five RCTs, which included 386 patients, to assess the effect of teriparatide on osteoporotic hip and pelvic bone fractures. Our revealed that there was no significant difference in the rates of treatment failure at the final follow-up between the teriparatide and control groups. This result suggested that there is a lack of 


\section{Hip \& Pelvis}

Nam Hoon Moon et al. Teriparatide and Bone Healing: Meta-analysis and Systematic Review

Table 2. Comparison of the Treatment Failure in Patients with Osteoporotic Hip and Pubic Bone Fracture between the Teriparatide and Placebo Group

\begin{tabular}{lccc}
\hline \hline Study lyear) & Teriparatide & Placebo & $P$-value \\
\hline Bhandari et al. ${ }^{18)}(2016)$ & $13 / 81(16.0)$ & $11 / 78(14.1)$ & 0.589 \\
Kanakaris et al. $^{27)}(2015)$ & $0 / 9(0)$ & $2 / 10(20.0)$ & 0.314 \\
Chesser et al. $^{201}(2016)$ & $0 / 15(0)$ & $0 / 14(0)$ & - \\
Aspenberg et al. ${ }^{19)}(2016)$ & $7 / 55(12.7)$ & $9 / 59(15.3)$ & 0.791 \\
Peichl et al. $^{26)}(2011)$ & $0 / 21(100)$ & $14 / 44(31.8)$ & 0.004 \\
\hline
\end{tabular}

values are presented as number of treatment failure/total number in the group (\%).

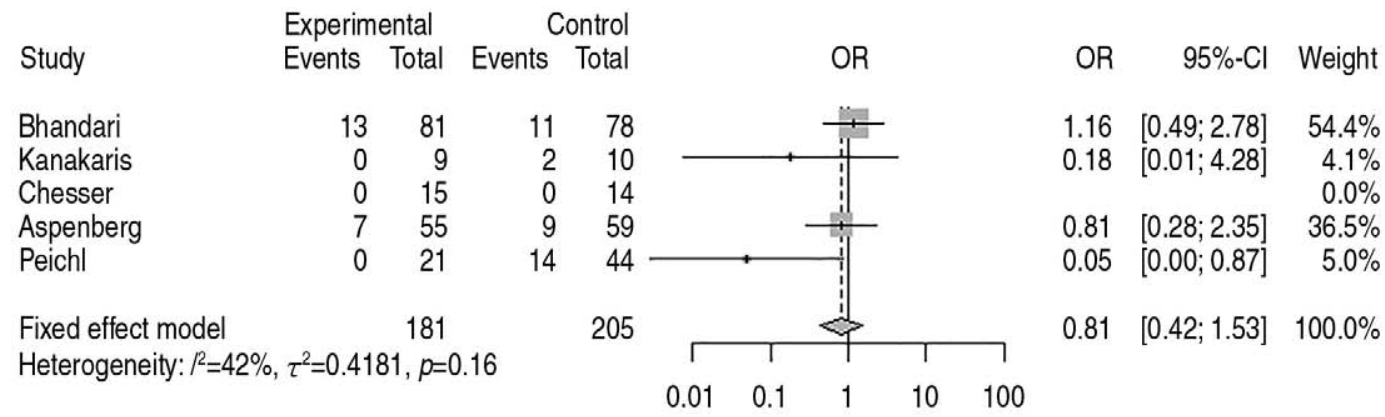

Fig. 2. Forest plot of the treatment outcomes.

OR: odds ratio, $\mathrm{Cl}$ : confidence interval.

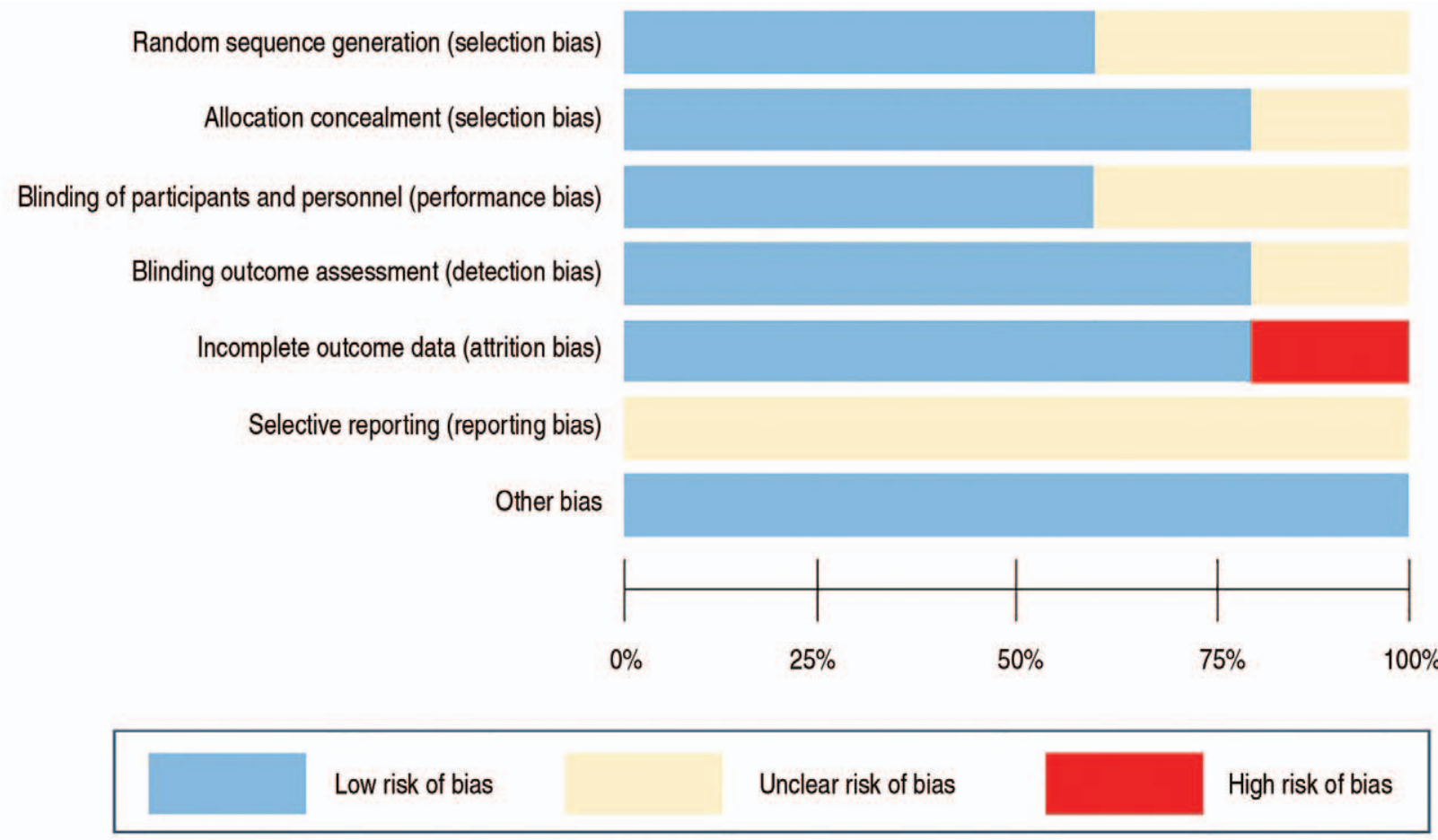

Fig. 3. Summary of risk of bias in the selected studies.

evidence demonstrating efficacy of teriparatide in treating osteoporotic hip and pelvic bone fractures, which was contrary to our hypothesis suggesting the clinical advantage of teriparatide.

Our study had several strengths. Firstly, our meta-analysis was compliant with the PRISMA guidelines and the recom- 


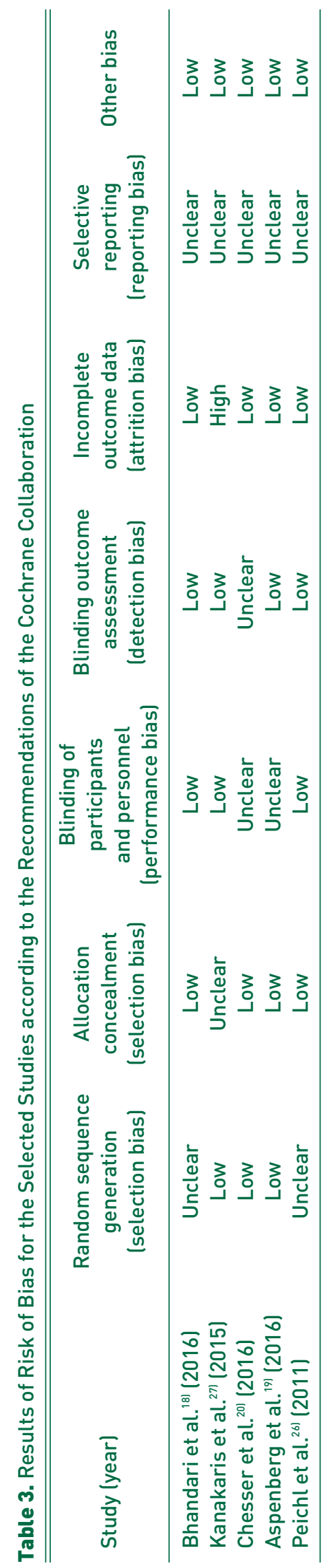

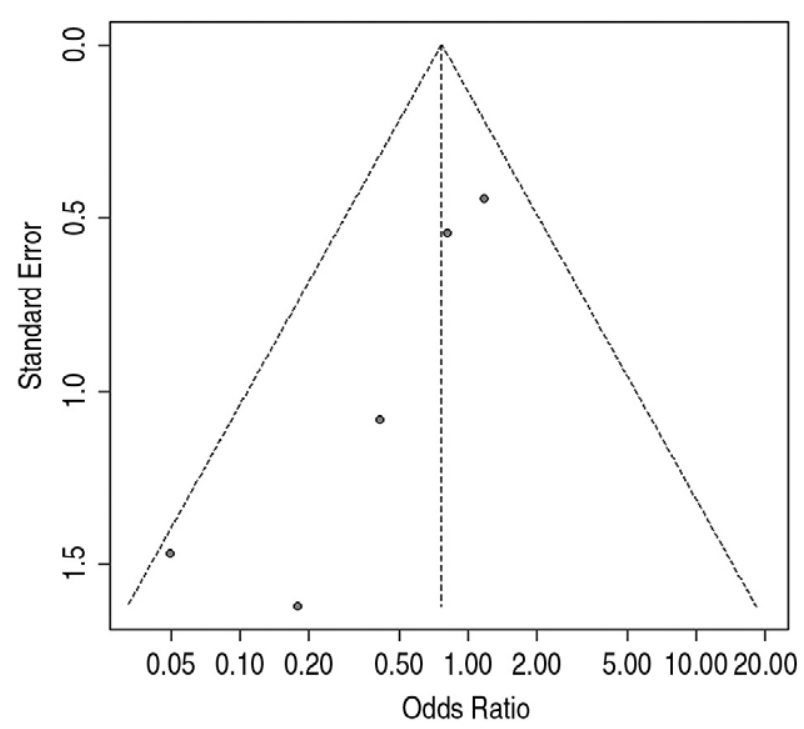

Fig. 4. Funnel plot for the treatment outcomes.

mendations of the Cochrane Collaboration. Secondly, the heterogeneity of enrolled studies was moderate $\left(\mathrm{I}^{2}=0.420\right)$. Previously published meta-analyses evaluating the effects of teriparatide on bone healing have included different bones including the humerus, radius, femur, spine, and pelvis, which resulted in a higher heterogeneity than reported here ${ }^{13,22,30,31)}$. As the process of bone healing and the effect of teriparatide can vary depending on mechanical stimulation including load-bearing, fracture healing can manifest differently in the upper and lower limbs and give rise to a higher heterogeneity $^{32,33}$. All previous studies indicated this factor as one of the major limitations of their respective studies, and consequently, the conclusions derived are inconsistent among studies. However, our meta-analysis included studies that focused exclusively on osteoporotic hip and pelvic bone fractures which, we believe, resulted in moderate heterogeneity. Thirdly, the hip and pelvic bones in elderly patients are most suitable to evaluate the effects of teriparatide on fracture healing. Osteoporotic hip and pelvic fractures are known to increase mortality owing to immobilization and subsequent medical complications (e.g., aspiration pneumonia, pressure sores, venous thromboembolism) ${ }^{2,23,24)}$. Thus, if the efficacy of teriparatide on treatment outcomes was established, this study could have supported the use of teriparatide in the treatment of osteoporotic hip and pelvic bone fractures. Unfortunately, our study did not demonstrate that teriparatide effectively improved treatment outcomes in these fractures. Therefore, this lack of evidence precludes the use of teriparatide to improve treatment outcomes in osteoporotic hip and pelvic bone fractures. 
Nam Hoon Moon et al. Teriparatide and Bone Healing: Meta-analysis and Systematic Review

There were a few limitations of this meta-analysis. Firstly, only five RCTs were included, which constitutes a relatively small sample size. Secondly, although we included RCTs involving osteoporotic hip and pelvic bone fractures, these studies also included various other fractures (e.g., femoral neck, intertrochanteric, subtrochanteric, pelvic bone), and their sub-classification could affect reported treatment outcomes. In particular, the study by Peichl et al. ${ }^{26)}$ exclusively included patients with pubic bone fractures, thus making it difficult to conclude that the results of the present study are representativeness of osteoporotic pelvic bone fractures. However, there have been no RCTs to date investigating insufficiency fractures of the pelvic bone related to osteoporosis, and we believe that osteoporotic pubic bone fracture is representative of insufficiency fractures of pelvic bone. Furthermore, various fracture-treatment protocols were identified including intramedullary nailing, fixation using a dynamic hip screw or cannulated screw, and conservative treatment, and there could have been additional factors that might have affected treatment outcomes including: treatment-related factors (e.g., surgical techniques, postoperative reduction), and patient-related factors (e.g., bone mineral density, body mass index, extent of preoperative mobility, rehabilitation compliance). Thirdly, each study had different treatment periods for teriparatide therapy and followups. Although teriparatide is known to induce early callus formation after fractures, the long-term bone-healing effect can vary depending on the treatment period, as teriparatide can not only affect the early bone-formation phase, but also the remodeling phase ${ }^{14,29,32}$. Additionally, some studies have demonstrated that functional improvement depends on the period of teriparatide treatment. Thus, it is important to consider studies with identical treatment periods when comparing clinical outcomes.

Next, this study could not assess other key clinical outcomes. The five studies included in our meta-analysis evaluated varying clinical outcomes that were not consistent across studies (e.g., Harris hip score, short form 12, short form 36, Timed Up and Go test, Johanson Hip Rating Questionnaire), thus making it difficult to comprehensively assess the clinical effect of teriparatide ${ }^{18,20,25-27,34)}$. Mortality after fracture is an important clinical outcome in the elderly with hip and pelvic bone fractures, however, none of the studies included in this meta-analysis evaluated mortality after fractures. In addition, this study did not include any data comparing the safety of teriparatide versus placebo groups. As the number of complications reported in the enrolled studies was not adequate to conduct statistical analysis, and considering that the side effects related directly to teriparatide were low, it was difficult to include safety data on teriparatide.

Lastly, the radiological outcomes of fracture healing evaluated in this study were limited. As the five studies selected for this meta-analysis included various radiological measurements at different time points and stages of follow-up, it was difficult to conduct a meta-analysis for the fracturehealing effect of teriparatide in the early stages of bone healing, including those after a 6- or 12-week follow-up. This was a major limitation of this study given that teriparatide increases callus formation at an early stage of bone healing. In particular, as the acceleration of bone healing in the early stage of osteoporotic hip and pelvic bone fractures can improve rehabilitation and lower long-term mortality rates, simply because there is no significant difference in treatment outcomes at the last follow-up does not mean that there are no positive effect of teriparatide on osteoporotic hip and pelvic bone fractures. Thus, further studies on the bone-healing effect of teriparatide in the early stages of healing are warranted.

\section{CONCLUSION}

This meta-analysis did not identify any significant differences in treatment failure rates at final follow-up between the teriparatide and control groups. Based on these results, we believe that there is a lack of evidence to suggest that teriparatide improves treatment failure rates in osteoporotic hip and pelvic fractures. However, further studies are needed to determine the efficacy of teriparatide on fracture healing and in improving other clinical outcomes.

\section{ACKNOWLEDGEMENTS}

This work was supported by a clinical research grant from Pusan National University Hospital in 2020.

\section{CONFLICT OF INTEREST}

The authors declare that there is no potential conflict of interest relevant to this article.

\section{REFERENCES}

1. Ha YC, Kim TY, Lee A, et al. Current trends and future projections of hip fracture in South Korea using nationwide claims data. Osteoporos Int. 2016;27:2603-9.

2. Ha YC, Park YG, Nam KW, Kim SR. Trend in hip fracture 
incidence and mortality in Korea: a prospective cohort study from 2002 to 2011. J Korean Med Sci. 2015;30:483-8.

3. Nakano T, Shiraki M, Sugimoto T, et al. Once-weekly teriparatide reduces the risk of vertebral fracture in patients with various fracture risks: subgroup analysis of the Teriparatide Once-Weekly Efficacy Research (TOWER) trial. J Bone Miner Metab. 2014;32:441-6.

4. Sugimoto T, Shiraki M, Nakano T, et al. Vertebral fracture risk after once-weekly teriparatide injections: follow-up study of Teriparatide Once-Weekly Efficacy Research (TOWER) trial. Curr Med Res Opin. 2013;29:195-203.

5. Nakamura T, Sugimoto T, Nakano T, et al. Randomized Teriparatide [human parathyroid hormone (PTH) 1-34] OnceWeekly Efficacy Research (TOWER) trial for examining the reduction in new vertebral fractures in subjects with primary osteoporosis and high fracture risk. J Clin Endocrinol Metab. 2012;97:3097-106.

6. Shiraki M, Ueda S, Sugimoto T, Kuroda T, Nakamura T. Treatment responses with once-weekly teriparatide therapy for osteoporosis. Osteoporos Int. 2016;27:3057-62.

7. Liu Y, Levack AE, Marty E, et al. Anabolic agents: what is beyond osteoporosis? Osteoporos Int. 2018;29:1009-22.

8. Lau EMC, Dinavahi R, Woo YC, et al. Romosozumab or alendronate for fracture prevention in East Asian patients: a subanalysis of the phase III, randomized ARCH study. Osteoporos Int. 2020;31:677-85.

9. Takada J, Dinavahi R, Miyauchi A, et al. Relationship between PINP, a biochemical marker of bone turnover, and bone mineral density in patients transitioned from alendronate to romosozumab or teriparatide: a post hoc analysis of the STRUCTURE trial. J Bone Miner Metab. 2020;38:310-5.

10. Kanezaki S, Miyazaki M, Ishihara T, et al. Enhancement of the effects of intermittent parathyroid hormone (1-34) by bone morphogenetic protein in a rat femoral open fracture model. J Orthop Surg Res. 2019;14:403.

11.Zandi M, Dehghan A, Bigonah N, Doulati S, Mohammad Gholi Mezerji N. Histological assessment of the effects of teriparatide therapy on mandibular fracture healing: a preclinical study. J Craniomaxillofac Surg. 2020;48:211-6.

12. Tsubouchi Y, Ikeda S, Kataoka M, Tsumura H. Combination therapy with low-dose teriparatide and zoledronate contributes to fracture healing on rat femoral fracture model. J Orthop Surg Res. 2018;13:267.

13. Shi Z, Zhou H, Pan B, et al. Effectiveness of teriparatide on fracture healing: a systematic review and meta-analysis. PLoS One. 2016;11:e0168691.

14. Shin WC, Moon NH, Jang JH, Seo HU, Suh KT. A retrospective bicenter comparative study of surgical outcomes of atypical femoral fracture: potential effect of teriparatide on fracture healing and callus formation. Bone. 2019;128:115033.

15. Yoo JI, Ha YC, Ryu HJ, et al. Teriparatide treatment in elderly patients with sacral insufficiency fracture. J Clin Endocrinol Metab. 2017;102:560-5.

16. Kim JT, Jeong HJ, Lee SJ, Kim HJ, Yoo JJ. Adjuvant teriparatide therapy for surgical treatment of femoral fractures; does it work? Hip Pelvis. 2016;28:148-56.

17. Oba H, Takahashi J, Yokomichi H, et al. Weekly teriparatide versus bisphosphonate for bone union during 6 months after multi-level lumbar interbody fusion for osteoporotic patients: a multicenter, prospective, randomized study. Spine (Phila Pa 1976). 2020;45:863-71.

18. Bhandari M, Jin L, See K, et al. Does teriparatide improve femoral neck fracture healing: results from a randomized placebo-controlled trial. Clin Orthop Relat Res. 2016;474: 1234-44.

19. Aspenberg P, Malouf J, Tarantino U, et al. Effects of teriparatide compared with risedronate on recovery after pertrochanteric hip fracture: results of a randomized, active-controlled, double-blind clinical trial at 26 weeks. J Bone Joint Surg Am. 2016;98:1868-78.

20. Chesser TJ, Fox R, Harding K, et al. The administration of intermittent parathyroid hormone affects functional recovery from trochanteric fractured neck of femur: a randomised prospective mixed method pilot study. Bone Joint J. 2016; 98:840-5.

21. Wang YK, Qin SQ, Ma T, et al. Effects of teriparatide versus alendronate for treatment of postmenopausal osteoporosis: a meta-analysis of randomized controlled trials. Medicine (Baltimore). 2017;96:e6970.

22. Yang C, Le G, Lu C, et al. Effects of teriparatide compared with risedronate in the treatment of osteoporosis: a metaanalysis of randomized controlled trials. Medicine (Baltimore). 2020;99:e 19042.

23. Sheehan KJ, Sobolev B, Guy P. Mortality by timing of hip fracture surgery: factors and relationships at play. J Bone Joint Surg Am. 2017;99:e106.

24. Katsoulis M, Benetou V, Karapetyan T, et al. Excess mortality after hip fracture in elderly persons from Europe and the USA: the CHANCES project. J Intern Med. 2017;281:300-10.

25. Huang TW, Chuang PY, Lin SJ, et al. Teriparatide improves fracture healing and early functional recovery in treatment of osteoporotic intertrochanteric fractures. Medicine (Baltimore). 2016;95:e3626.

26. Peichl P, Holzer LA, Maier R, Holzer G. Parathyroid hormone 1-84 accelerates fracture-healing in pubic bones of elderly osteoporotic women. J Bone Joint Surg Am. 2011;93:1583-7.

27. Kanakaris NK, West RM, Giannoudis PV. Enhancement of hip fracture healing in the elderly: evidence deriving from $a$ pilot randomized trial. Injury. 2015;46:1425-8.

28. Higgins JPT, Green S. Cochrane handbook for systematic reviews of interventions: version 5.1.0 [Internet]. London: The Cochrane Collaboration; 2011 Mar 20 [cited 2019 Jul 1]. Available from: https://handbook-5-1.cochrane.org/.

29. Rhee SJ, Kim HJ, Lee CR, Kim CW, Gwak HC, Kim JH. A comparison of long-term outcomes of computer-navigated and conventional total knee arthroplasty: a meta-analysis of randomized controlled trials. J Bone Joint Surg Am. 2019; 101:1875-85.

30. Lou S, Lv H, Li Z, Tang P, Wang Y. Parathyroid hormone analogues for fracture healing: protocol for a systematic review and meta-analysis of randomised controlled trials. BMJ Open. 2018;8:e019291.

31. Lou S, Lv H, Wang G, et al. The effect of teriparatide on fracture healing of osteoporotic patients: a meta-analysis of randomized controlled trials. Biomed Res Int. 2016;2016: 6040379.

32. Díez-Pérez A, Marin F, Eriksen EF, Kendler DL, Krege JH, Delgado-Rodríguez M. Effects of teriparatide on hip and 


\section{Hip \& Pelvis}

Nam Hoon Moon et al. Teriparatide and Bone Healing: Meta-analysis and Systematic Review

upper limb fractures in patients with osteoporosis: a systematic review and meta-analysis. Bone. 2019;120:1-8.

33. Johansson T. PTH 1-34 (teriparatide) may not improve healing in proximal humerus fractures. A randomized, con- trolled study of 40 patients. Acta Orthop. 2016;87:79-82.

34. Aspenberg P, Johansson T. Teriparatide improves early callus formation in distal radial fractures. Acta Orthop. 2010; 81:234-6. 